\title{
Describing Nuclear Matter with Effective Field Theories
}

\author{
James V. Steele and R.J. Furnstahla* \\ aDepartment of Physics, The Ohio State University, Columbus, OH 43210 USA
}

An accurate description of nuclear matter starting from free-space nuclear forces has been an elusive goal. The complexity of the system makes approximations inevitable, so the challenge is to find a consistent truncation scheme with controlled errors. The virtues of an effective field theory approach to this problem are discussed.

Nuclear forces have been studied in depth over the past fifty years, leading to excellent phenomenological descriptions. A recent resurgence of interest in this field has been fueled by the promise of systematic results from an effective field theory (EFT) analysis [1]. EFT techniques adapted to many-body systems may give new insight into the properties of nuclei and their connection to QCD.

The EFT lagrangian consists of long-range interactions constrained by chiral symmetry and the most general short-range interactions consistent with QCD symmetries. The coefficients of these short-range terms may eventually be derived from QCD, but at present must be fit by matching calculated and experimental observables in a momentum expansion. This effective lagrangian can then be used to systematically predict other observables, including inelastic processes.

The predictability of an EFT relies on an organizational scheme, called power counting, to determine the importance of any contribution to a calculation. This can be illustrated by using the familiar one-boson-exchange representation of the NN force as a model of the true underlying physics. One-pion exchange (OPE) is taken to be the long-range physics, as its contribution to the EFT is dictated by chiral symmetry. Exchanges of the heavier mesons will be considered short-range physics, which must be included generically in the EFT. For example, for center-of-mass momenta below the sigma mass, $p<m_{\sigma}$, the heavy-meson propagators are well approximated by momentum-dependent contact interactions,

$\frac{1}{p^{2}-m_{\sigma}^{2}}=C_{0}+C_{2} p^{2}+C_{4} p^{4}+\ldots$

with $C_{2}=C_{0} / m_{\sigma}^{2}$ and so on. Each term has an associated power of $p / m_{\sigma}$, which carries over to observables. A consistent truncation based on counting these powers leads to observables with well-defined errors [1]. Here $m_{\sigma}$ plays the role of the EFT breakdown scale $\Lambda$. At momenta of order $\Lambda$, the short-distance structure is resolved, all higher-order corrections become comparable in magnitude, and consequently the EFT fails.

${ }^{*}$ Supported in part by NSF grants PHY-9511923 and PHY-9800964. 


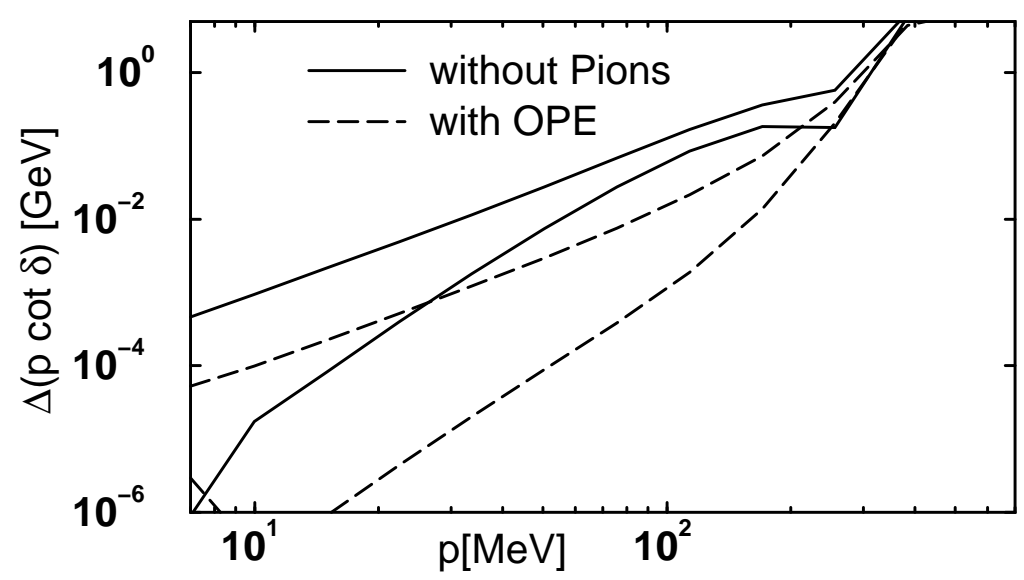

Figure 1. The error in $p \cot \delta$ for ${ }^{1} S_{0} n p$ scattering using cutoff regularization without pions (solid) and with one-pion exchange (dashed), both shown for one and two constants [1].

In the early years of research into nuclear forces, Bethe showed that low-energy scattering between two nucleons could be expressed in a form now known as the effective range expansion. For example, in the ${ }^{1} S_{0}$ channel,

$p \cot \delta=-\frac{1}{a_{s}}+\frac{1}{2} r_{e} p^{2}+\ldots$.

A key observation is that the coefficients $a_{s}, r_{e}, \ldots$, of this momentum expansion are independent of the details of the potential used to fit low-energy data. The scale associated with the parameters is the point at which Eq. (2) breaks down, around $p \sim 1 / r_{e} \sim m_{\pi}$. This expansion is suggestive of an EFT with a breakdown scale around $\Lambda \sim m_{\pi}$. So we expect to reproduce Eq. (2) if we treat all interactions (including OPE) as short-ranged.

Due to the existence of nuclear bound states (such as the deuteron) near threshold, an EFT analysis for multiple nucleons requires a nonperturbative treatment. One formulation is based on the Lippmann-Schwinger equation for the $T$-matrix [1], $\hat{T}=\hat{V}+\hat{V} \hat{G}_{0} \hat{T}$. The most general effective potential with no long-range physics takes the form

$\hat{V}_{\mathrm{EFT}}=C_{0}+C_{2} \hat{p}^{2}+\mathcal{O}\left(\hat{p}^{4} / \Lambda^{4}\right)$.

If the EFT phase shifts are calculated and compared with actual data, we find that the constants indeed form a hierarchy analogous to Eq. (1), but with the relevant scale being set by the pion mass $m_{\pi}$ :

$C_{0} \sim \frac{4 \pi}{M m_{\pi}}, \quad C_{2} p^{2} \sim \frac{4 \pi}{M m_{\pi}} \frac{p^{2}}{m_{\pi}^{2}}, \quad \ldots$.

Enforcing this power counting order-by-order in $p^{2}$ reproduces the effective range expansion Eq. (2) and generates the solid lines in the error plot of Fig. 11. The error is dominated by the first omitted term and so behaves like a power of $p^{2}$. As more contact interactions are included in the effective potential Eq. (3), the slope of the error curves gets steeper. The point at which the linear parts of the curves converge [4] indicates the breakdown scale $\Lambda$, which is indeed of order $m_{\pi}$ (actually $m_{\pi} / 2$ ).

To push beyond this scale, pions must be explicitly included in the effective lagrangian as long-range physics. There are alternative power counting schemes proposed in the 
literature [1]. In Fig. 1, results are shown for a scheme in which one-pion exchange enters at leading order and pion effects are treated nonperturbatively. The range of validity of the EFT is extended to about $\Lambda=300 \mathrm{MeV}$ in that case, as seen by the dashed lines.

A complete calculation at next-to-leading order requires irreducible two-pion exchange. Preliminary results suggest that with a careful removal of long-distance contamination from coefficients by using a modified effective range expansion [ [1], the EFT range of validity may extend as high as $\Lambda \sim m_{\rho}$ [6]. Even then, the accuracy of the EFT results for NN scattering is not yet competitive with that of the Bonn potential [6]. Nevertheless, the error plots manifest a systematic expansion that allows realistic error estimates for scattering and other observables, which are not available in conventional approaches.

How does this expansion and breakdown scale carry over to nuclear matter? The complications of the many-body problem can obscure the connection, but we can use a perturbative matching calculation to understand the general correspondence. To proceed, model data can be generated from an exactly solvable potential with weak coupling $\lambda$. The EFT contact interactions is then fit by this data up to a given order in $\lambda$; for example,

$\hat{T}_{\mathrm{EFT}}=\hat{V}_{\mathrm{EFT}}+\hat{V}_{\mathrm{EFT}} \hat{G}_{0} \hat{V}_{\mathrm{EFT}}+\mathcal{O}\left(\lambda^{3}\right)$.

The regularization of divergences in the second term of Eq. (5) necessitates a renormalization of the first term to ensure a cutoff independent match. Solving for the exact in-medium $T$-matrix $\hat{\Gamma}$ perturbatively and comparing with the EFT result using the effective potential in Eq. (3) verifies that the match remains regulator independent to the same order as in free space,

$\hat{\Gamma}=\hat{\Gamma}_{\mathrm{EFT}}+\mathcal{O}\left(\lambda^{3}\right)+\mathcal{O}\left(k_{F}^{4} / \Lambda^{4}\right)$.

The truncation error from the EFT expansion in nuclear matter is a power of the Fermi momentum over the free-space breakdown scale, $k_{F} / \Lambda$ [ 8]. This is very encouraging, since phenomenologically successful mean-field descriptions have a related expansion with $\Lambda \simeq 600 \mathrm{MeV}$ [7]. Thus if the free-space breakdown scale is indeed as large as $\Lambda \sim m_{\rho}$, a useful EFT expansion of nuclear matter is likely.

A nonperturbative EFT calculation of nuclear matter introduces many complications compared to the free-space EFT. The analogue of scattering between two nucleons involves particles propagating off the energy-shell $\left(E \neq p^{2} / M\right)$ and the in-medium $T$-matrix not only depends on the relative momentum, but also the total momentum. Also, enforcing the Pauli exclusion principle greatly complicates the momentum integrals. However, a systematic EFT error analysis makes the calculation worth the effort, and the EFT approach can also provide new perspectives on old issues and practices. Some examples: i) Nuclear saturation with velocity-dependent potentials instead of a hard core; ii) Renormalization scheme independence of observables, such as the binding energy, requires the sum of ladder diagrams, providing a new justification for this approximation; iii) A self-consistent ladder calculation requires explicit three-body forces. This last feature might account for discrepancies in nuclear matter between phase-equivalent two-body potentials [7].

To see the necessity of three-body forces, consider three-to-three scattering with only two-body interactions, as depicted by the second Feynman diagram in Fig. 2. This could arise from the first diagram, which is a contribution to the energy of the nuclear matter ground state, by expanding the short-range interactions in contact terms and opening the 


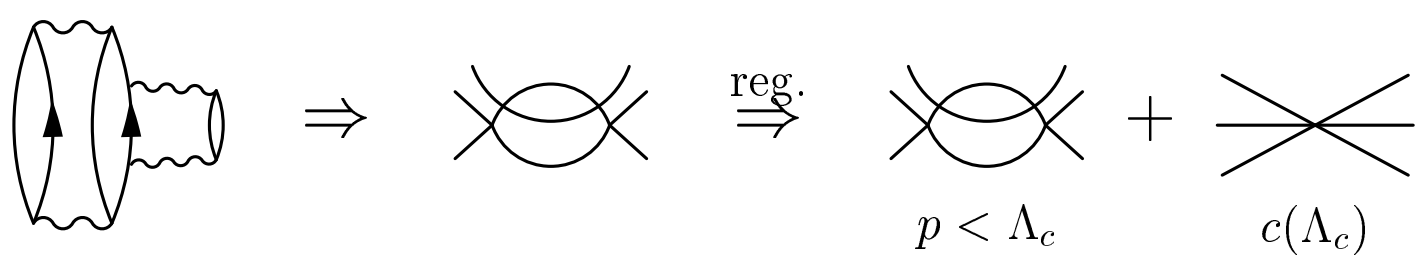

Figure 2. Regularization of a fourth-order contribution to the energy featuring only twobody interactions requires three-body contact interactions in the EFT.

hole lines. Since the EFT is only accurate at low momenta, the divergent loop integrals should be cut off at some scale $\Lambda_{c}$. The intermediate states above $\Lambda_{c}$ are highly virtual, and so by the uncertainty principle are well represented by a regularized series of threebody contact interactions. These interactions are needed to guarantee regularization independence of the final result and to account for contributions from the suppressed physical degrees of freedom.

Higher-body interactions are cut off in a similar fashion, implying an infinite number of constants and many-body forces would be needed to describe nuclear matter. The Pauli exclusion principle helps the situation by limiting the number of contact interactions with no derivatives to four nucleons or fewer, and the EFT power counting implies that other higher-body interactions between nucleons are suppressed. However, the nature of the power counting is still under investigation, and it is possible that three- and four-body contact terms are needed even at leading order [2] in a nuclear matter calculation.

The inevitability of many-body forces in the EFT analysis highlights the fact that the potential is not an observable. There is no such thing as a "best" two-body potential and differences in two-body off-shell behavior in nuclear matter may be compensated by many-body counterterms [7]. In the case of three-nucleon scattering, accounting for the three-body contact interaction leads to a compelling explanation of the Phillips line [2]. An interesting possibility is that an extension of this analysis to nuclear matter could explain the Coester line [3].

While the application of effective field theory to nuclear matter is in its infancy, systematic EFT calculations could help settle long-standing issues, justify traditional expansions, or even offer new alternatives. In the long run, EFT techniques lay a foundation for ultimately connecting the physics of nuclei to QCD.

\section{REFERENCES}

1. Proceedings of the Joint Caltech/INT Workshop: Nuclear Physics with Effective Field Theory, ed. R. Seki, U. van Kolck, and M.J. Savage (World Scientific, 1998).

2. P.F. Bedaque, H.-W. Hammer, and U. van Kolck, Nucl. Phys. A646 (1999) 444.

3. R. Machleidt, Adv. Nucl. Phys. 19 (1989) 189, and references therein.

4. J.V. Steele and R.J. Furnstahl, Nucl. Phys. A637 (1998) 46; A645 (1999) 439.

5. D.B. Kaplan, M.J. Savage, and M.B. Wise, Nucl. Phys. B534 (1998) 329.

6. J.V. Steele and R.J. Furnstahl, in preparation.

7. R.J. Furnstahl, J.V. Steele, and N. Tirfessa, in preparation.

8. M. Lutz, B. Friman, and C. Appel, nucl-th/9907078. 